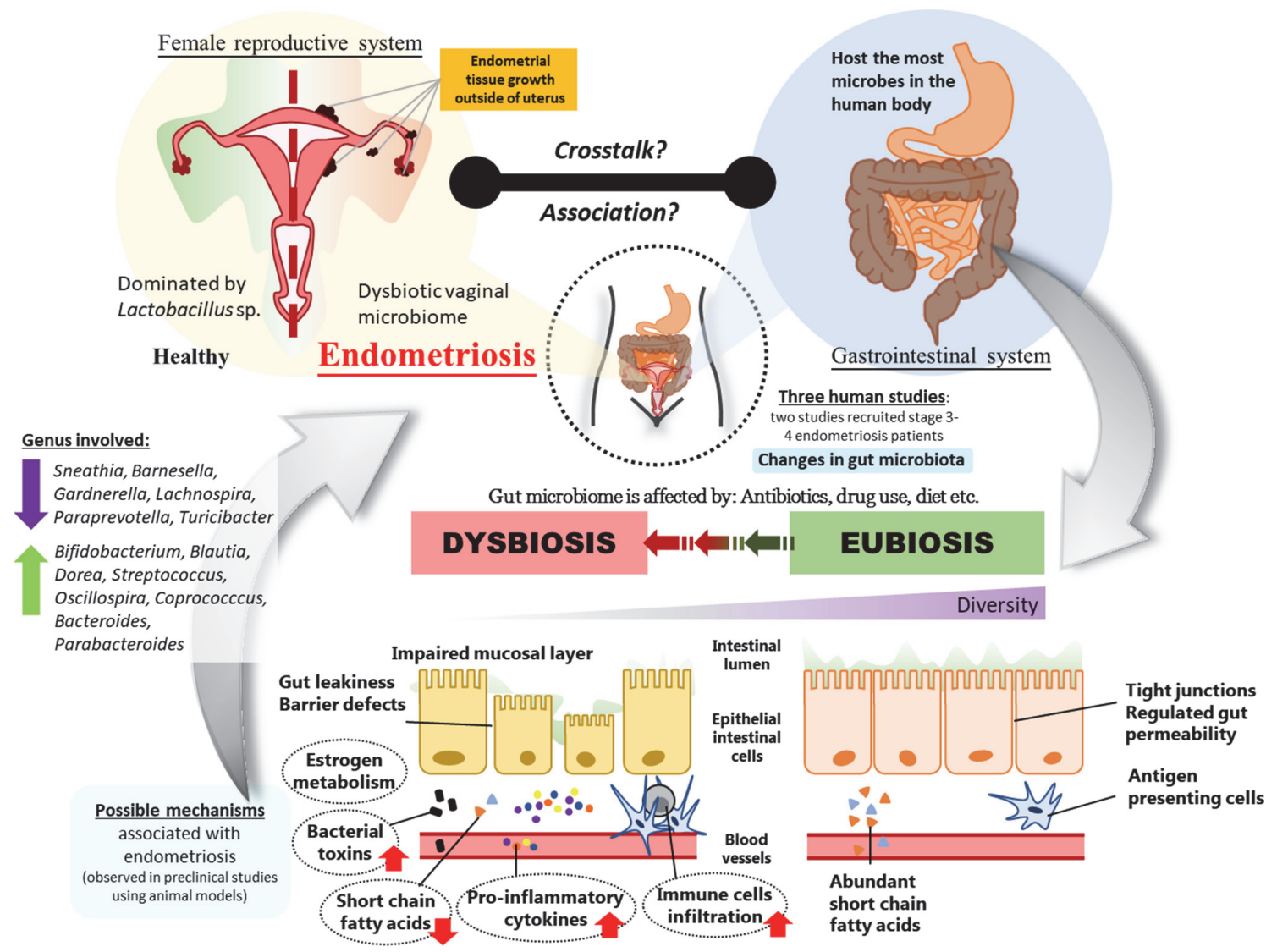

Abstract IDDF2021-ABS-0132 Figure 1

titles and abstracts retrieved were screened based on the inclusion and exclusion criteria. Studies reporting gut microbiome data in relation to endometriosis in humans were included in the current analysis but not those without gut microbiome data.

Results Three studies were selected for qualitative analysis according to the inclusion and exclusion criteria. All studies investigated the gut microbiota composition by whole genome sequencing, specifically targeting the $16 \mathrm{~S}$ rRNA gene. These studies detected significant differences in selected bacterial abundance in the gut microbiome among endometriosis patients and control. Two of them discussed potential bacterial group(s) that correlates with gastrointestinal symptoms in endometriosis patients. A study in 2019 showed that two endometriosis patients had high Escherichia/Shigella in stool, and subsequent follow-up showed severe bowel involvement by endometriosis requiring segmental colon resection. Another team also indicated in their recent work that those with endometrial involvement of the gastrointestinal tract had a higher abundance of Lactococcus (Class Bacilli) compared to those without involvement. The same study noted a non-significant enrichment of Enterobacteriaceae in endometriosis patients that warrants further investigation to evaluate the involvement of gut microbiota in endometriosis.

Conclusions Further studies are required to establish the association and involvement of gut microbiota in endometriosis. The heterogeneity in study design (inclusion/exclusion criteria etc.) increases the difficulty in making a meaningful comparison. More information on diet and antibiotic drug use need to be collected to provide a better overview and insightful analysis of the gut microbiome in endometriosis.

\section{IDDF2021-ABS-0140 GUT MICROBIOTA SIGNIFICANTLY CORRELATE WITH BODY CONSTITUTION IN TRADITIONAL CHINESE MEDICINE}

${ }^{1}$ Haohui Liu*, ${ }^{2}$ Meihui Xu, ${ }^{2}$ Kai Yee Toh, ${ }^{3}$ Chun Wie Chong, ${ }^{2}$ Jeremy Fung Yen Lim, ${ }^{4} J$ onathan Wei Jie Lee. ${ }^{1}$ School of Computer Science, Carnegie Mellon University, USA; ${ }^{2}$ Asian Microbiome Library (AMILI), Singapore; ${ }^{3}$ School of Pharmacy, Monash University Malaysia, Malaysia; ${ }^{4}$ Division of Gastroenterology and Hepatology, National University Hospital, Singapore

\subsection{6/gutjil-2021-IDDF.45}

Background The gut microbiome plays an instrumental role in cardiovascular, metabolic and gastrointestinal health (Valdes et al. 2018). In Traditional Chinese Medicine (TCM), one's health status can be described by nine body constitutions (BCs) which are broadly classified as 'Neutral', indicating optimal health, or 'Unbalanced', indicating suboptimal health. Similar to the gut microbiome, BCs are strongly influenced by lifestyle and diet. We evaluated the gut microbial composition of Neutral, Qi-Deficiency (QD), Phlegm-dampness (PD) and Damp Heat (DH) subjects. The latter three are unbalanced BCs, with QD associated with heart disease (Zhu et al. 2017), PD with obesity and metabolic syndrome (Wang et al. 2013), and DH with gastrointestinal disease (Liang et al. 2020).

Methods Stool samples were collected from 211 healthy subjects and gut microbial composition was profiled using $16 \mathrm{~S}$ rRNA amplicon sequencing of the V3-V4 regions. BCs were determined using the Constitution in Chinese Medicine Questionnaire (Wang, 2005). Alpha diversity of QD, PD and DH was inferred and compared against that of Neutral using the 


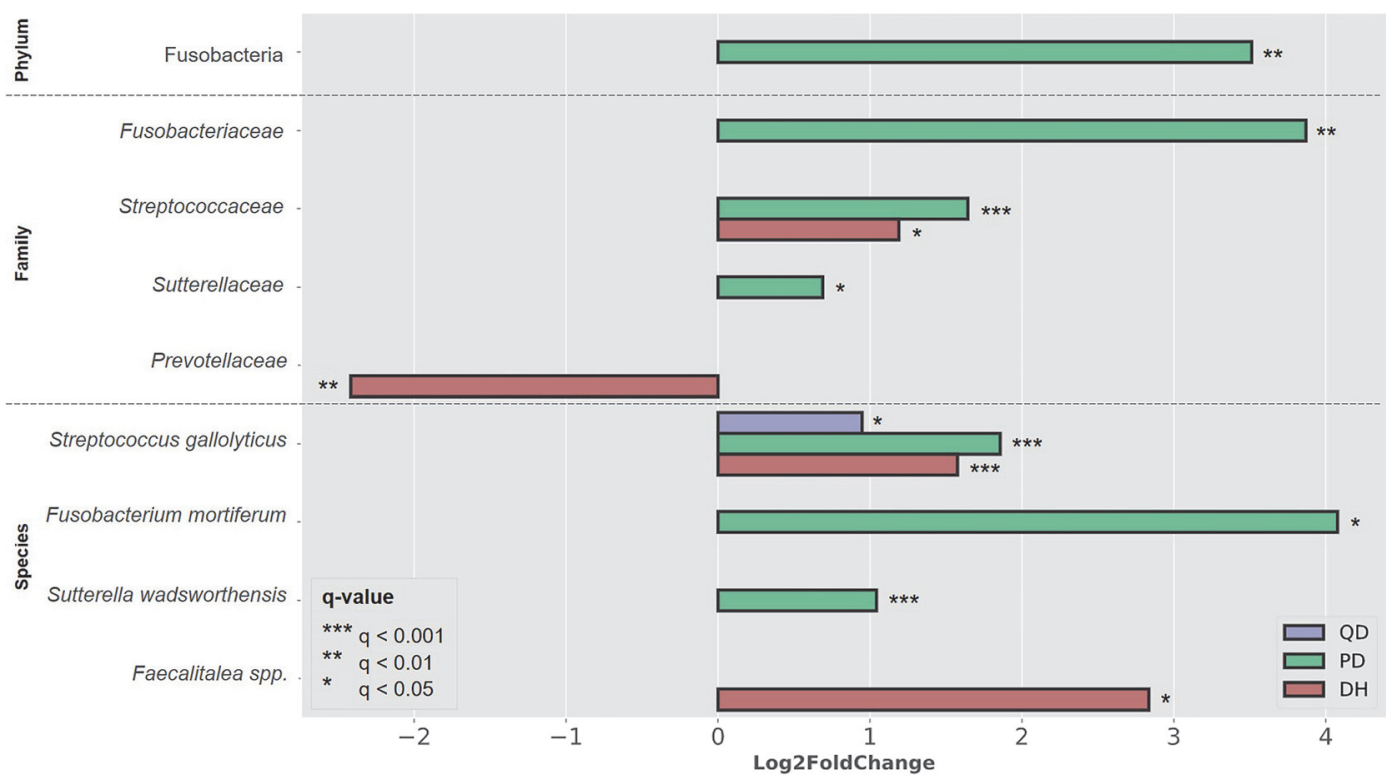

\section{Abstract IDDF2021-ABS-0140 Figure 1}

Kruskal-Wallis test. Differentially abundant bacterial phyla, family and species were identified by calculating Log2FoldChange of taxa in the unbalanced BCs compared to Neutral. Results There were 135 Neutral, 59 QD, 24 PD and $12 \mathrm{DH}$ subjects. 16 participants exhibited two or more unbalanced BCs. PD exhibited significantly lower alpha diversity (Shannon, Simpson) than Neutral $(\mathrm{p}<0.05)$. Fusobacteria (phylum) was elevated in PD $(p<0.01)$. At the species level, Streptococcus gallolyticus was significantly higher in all three unbalanced BCs relative to Neutral. Fusobacterium mortiferum and Sutterella wadsworthensis were higher in PD than Neutral. Faecalitalea spp. was higher in DH than Neutral (IDDF2021-ABS-0140 Figure 1. Log2FoldChange of differentially abundant bacteria in $\mathrm{QD}, \mathrm{PD}$ and $\mathrm{DH}$ as compared to Neutral).

Conclusions Varying TCM BCs exhibit unique microbiota signatures. Participants with unbalanced BCs are associated with greater dysbiosis compared to participants with Neutral BC. The lower alpha diversity in PD is consistent with that in obese and metabolic syndrome patients (Stanislawski et al. 2019; Oh et al. 2021). Streptococcus gallolyticus, a species associated with gastrointestinal malignancy (Chand et al. 2016), was significantly higher in all three unbalanced BCs and thus may serve as a potential biomarker for identifying patients with unbalanced BCs.

\section{IDDF2021-ABS-0150 INFLUENCE OF ETHNICITY ON THE GUT MICROBIOTA OF SINGAPOREAN AND MALAYSIAN COMMUNITIES}

${ }^{1}$ Jacky Dwiyanto*, ${ }^{2}$ Kai Yee Toh, ${ }^{2}$ Jia Pei Ho, ${ }^{3}$ Tin Tin Su, ${ }^{2}$ Jeremy Lim, ${ }^{4}$ Jonathan Wei Jie Lee, ${ }^{5}$ Sadequr Rahman, ${ }^{6}$ Chun Wie Chong. ${ }^{1}$ School of Science, Monash University Malaysia, Malaysia; ${ }^{2}$ Asian Microbiome Library (AMILI), Singapore; ${ }^{3}$ South East Asia Community Observatory (SEACO), Monash University Malaysia, Malaysia; ${ }^{4}$ Department of Gastroenterology and Hepatology, National University Hospital, Singapore; ${ }^{5}$ Tropical Medicine and Biology, Monash University Malaysia, Malaysia; ${ }^{6}$ School of Pharmacy, Monash University Malaysia, Malaysia

10.1136/gutjnl-2021-IDDF.46

Background Singapore and Malaysia are neighbouring countries with similar ethnic make-up: Chinese, Indian, and
Malays. However, Singapore is a high-income economy while Malaysia is a middle-income economy. Such a difference has resulted in some levels of dichotomy in diet and lifestyles. In this study, we sought to investigate the gut-ethnic variation across the two neighbouring countries with varying levels of economic development.

Methods A total of 439 relatively healthy Malaysian $(n=190)$ and Singaporean $(\mathrm{n}=249)$ adults $(>18)$ were included, comprising Chinese $(n=240)$, Indian $(n=74)$, Malay $(n=40)$, and the indigenous Jakun community $(n=85)$. The sequences were processed with DADA2 and annotated using the SILVA database.

Results Country of origin explained the most variation in the gut microbiota (PERMANOVA, Pseudo-F $=80.798$, $\mathrm{R}^{2}=0.156, \mathrm{p}=0.001$; IDDF2021-ABS-0150 Figure 1. Ordination). Importantly, ethnicity was still significantly

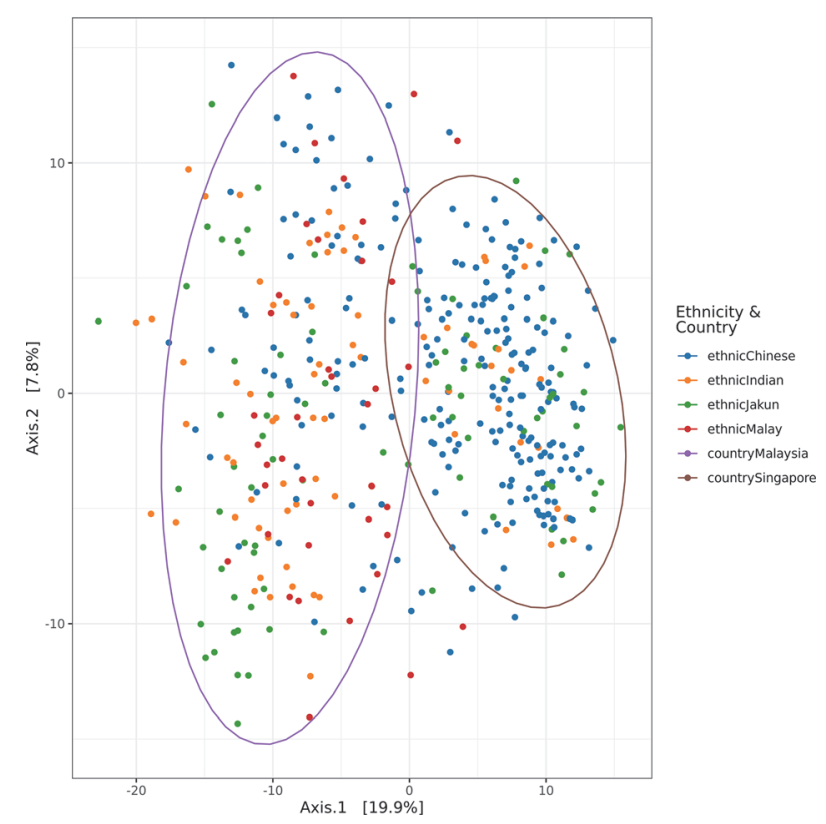

Abstract IDDF2021-ABS-0150 Figure 1 